\title{
Light depolarization by inhomogeneous linear birefringent media
}

\author{
S.N. Savenkov, Y.A. Oberemok, V.V. Yakubchak, Y.V. Aulin, O.I. Barchuk \\ Taras Shevchenko Kyiv National University, Radiophysics Department, \\ 5 build., 2, Acad. Glushkov ave., 03127 Kyiv, Ukraine, \\ phone: (380-44)526-04-83; e-mail: sns@univ.kiev.ua
}

\begin{abstract}
The purpose of the article is to provide rigorous analysis of light depolarization by inhomogeneous linear birefringent media in single scattering case. The object under investigation is a lossless anisotropic crystalline slab with surface inhomogeneity. For the analysis we use the Mueller matrix model of such class of media derived in [1], Cloude's coherency matrix method and known single value depolarization metrics. Sample calculations are given for calcite $\mathrm{CaCO}_{3}$, paratellurite $\mathrm{TeO}_{2}$ and lithium niobate $\mathrm{LiNbO}_{3}$.
\end{abstract}

Keywords: light scattering, depolarization, Mueller matrix, birefringence, Cloude's coherency matrix, entropy.

Manuscript received 09.12.08; accepted for publication 18.12.08; published online 02.03.09.

\section{Introduction}

When the polarization state of the light is characterized by means of the Stokes parameters, the transformation matrix is known as the Mueller matrix [2, 3]. From the properties of the Mueller matrix, one can draw insightful information about the underlying system. The exploitation of light polarization properties has a wide range of applications in a variety of fields, namely photonics technology [2, 3], astrophysics [4], biological and ecological optics [5] where scattering is linked to polarization state transformation and occurrence of depolarization. Depolarization is the result of decorrelation of the phases and amplitudes of the light electric vector and selective absorption of polarization states [2].

As shown earlier [1], the Mueller matrix of anisotropic lossless crystalline slab with surface inhomogeneity (roughness) in the eigen coordinate system has the following form:

$\mathbf{M}=\left(\begin{array}{cccc}\Phi_{11}+\Phi_{22} & \Phi_{11}-\Phi_{22} & 0 & 0 \\ \Phi_{11}-\Phi_{22} & \Phi_{11}+\Phi_{22} & 0 & 0 \\ 0 & 0 & \Phi_{12}+\Phi_{21} & i\left(\Phi_{12}-\Phi_{21}\right) \\ 0 & 0 & -i\left(\Phi_{12}-\Phi_{21}\right) & \Phi_{12}+\Phi_{21}\end{array}\right)$,

where

$$
\begin{aligned}
& \Phi_{x y}=\Phi_{x y}^{b r f}\left(\left(1-\eta_{x y}\right) \exp \left\{-\left(\frac{k \rho w}{2 z}\right)^{2}\right\}+\right. \\
& +\frac{\eta_{x y}}{\sigma_{x y}^{2} w^{2}+1} \exp \left\{-\frac{1}{\sigma_{x y}^{2} w^{2}+1}\left(\frac{k \rho w}{2 z}\right)^{2}\right\},
\end{aligned}
$$

$$
\begin{aligned}
& \Phi_{x y}^{b r f}=\exp \left(i k\left(n_{o}-n_{e}\right) \bar{h}-k^{2} \sigma_{h}^{2}\left(n_{o}-n_{e}\right)^{2} / 2\right), \\
& w=d\left(1+4 z^{2} / d^{4} k^{2}\right)^{1 / 2}, \\
& \sigma_{x y}^{2}=k^{2} \sigma_{h}^{2}\left(n_{o}-1\right)\left(n_{e}-1\right), \\
& \eta_{x y}=1-\exp \left(-\sigma_{x y}^{2}\right),
\end{aligned}
$$

$k=2 \pi / \lambda$ is a wavenumber; $\bar{h}$ is the thickness of a crystalline slab; $n_{o, e}$ denotes refractive indexes of medium associated with its linear eigenpolarizations, and $d$ is the beam radius.

After performing normalization, we get:

$\mathbf{M}=\left(\begin{array}{cccc}1 & a & 0 & 0 \\ a & 1 & 0 & 0 \\ 0 & 0 & b \cos (\psi) & b \sin (\psi) \\ 0 & 0 & -b \sin (\psi) & b \cos (\psi)\end{array}\right)$,

where

$a=\left(\Phi_{11}-\Phi_{22}\right) /\left(\Phi_{11}+\Phi_{22}\right)$,

$b=2\left|\Phi_{12}\right| /\left(\Phi_{11}+\Phi_{22}\right)$,

$\psi=\arg \left(\Phi_{12}^{b r f}\right)$.

To describe depolarization, it has been introduced several the so-called "single value depolarization metrics". In this paper, we use only those of them which do not need scanning the polarization states of input light for characterization of medium depolarization ability. First single value metrics is the depolarization index DI introduced in [6]: 
$D I=\left(\sum_{i, j=1}^{4} m_{i j}^{2}-m_{11}^{2}\right)^{\frac{1}{2}} / \sqrt{3} m_{11}$.

where $m_{i j}$ are Mueller matrix elements in the eigen coordinate system. The depolarization index lies within the range $0 \leq D I \leq 1$. Boundary values of $D I$ associate with the case of unpolarized and totally polarized output light, respectively.

The $Q$-metrics is [7]:

$Q=\sum_{\substack{i=2 \\ j=1}}^{4} m_{i j}^{2} / \sum_{j=1}^{4} m_{1 j}^{2}=\frac{3[D I]^{2}-[D]^{2}}{1+[D]^{2}}$,

where $D=\sqrt{m_{12}^{2}+m_{13}^{2}+m_{14}^{2}}$ is the diattenuation parameter and $0 \leq D \leq 1$.

The bound on the $Q$-metric is $0 \leq Q \leq 3$. In this case, $Q=0$ corresponds to totally depolarizing medium; $0<Q<1-$ to partially depolarizing medium; $1 \leq Q<3$ represents a partially depolarizing medium if, in addition, $0<D I<1$, otherwise, represents nondepolarizing diattenuating medium; $Q=3$ is for nondepolarizing non-diattenuating medium.

One more singlular value depolarization metric that we use below is entropy. The entropy $S$ was introduced in Cloude's coherency matrix method $[8,9]$, which is as follows. The coherence matrix $\mathbf{J}$ (with elements $J_{i j}$ ) is derived from the corresponding Mueller matrix as:

$$
\begin{aligned}
& J_{11}=1 / 4\left(m_{11}+m_{22}+m_{33}+m_{44}\right) \\
& J_{13}=1 / 4\left(m_{13}+m_{31}+i m_{24}-i m_{42}\right) \\
& J_{21}=1 / 4\left(m_{12}+m_{21}+i m_{34}-i m_{43}\right) \\
& J_{23}=1 / 4\left(i m_{14}+m_{23}+m_{32}-i m_{41}\right) \\
& J_{31}=1 / 4\left(m_{13}+m_{31}-i m_{24}+i m_{42}\right) \\
& J_{33}=1 / 4\left(m_{11}-m_{22}+m_{33}-m_{44}\right) \\
& J_{41}=1 / 4\left(m_{14}+i m_{23}-i m_{32}+m_{41}\right) \\
& J_{43}=1 / 4\left(-i m_{12}+i m_{21}+m_{34}+m_{43}\right) \\
& J_{12}=1 / 4\left(m_{12}+m_{21}-i m_{34}+i m_{43}\right) \\
& J_{14}=1 / 4\left(m_{14}-i m_{23}+i m_{32}+m_{41}\right) \\
& J_{22}=1 / 4\left(m_{11}+m_{22}-m_{33}-m_{44}\right) \\
& J_{24}=1 / 4\left(-i m_{13}+i m_{31}+m_{24}+m_{42}\right) \\
& J_{32}=1 / 4\left(-i m_{14}+m_{23}+m_{32}+i m_{41}\right) \\
& J_{34}=1 / 4\left(i m_{12}-i m_{21}+m_{34}+m_{43}\right) \\
& J_{42}=1 / 4\left(i m_{13}-i m_{31}+m_{24}+m_{42}\right) \\
& J_{44}=1 / 4\left(m_{11}-m_{22}-m_{33}+m_{44}\right) .
\end{aligned}
$$

Thus, $\mathbf{J}$ depends linearly on $\mathbf{M}$. It can be seen that the coherence matrix $\mathbf{J}$ is positive semidefinite Hermitian and, hence, has always four real eigenvalues. The eigenvalues of the coherence matrix, $\lambda_{i}$, can be combined to form a quantity that is a measure of depolarization in the studied medium. This quantity is called entropy and is defined as:

$S=-\sum_{i=1}^{N}\left(\lambda_{i} / \sum_{j} \lambda_{j}\right) \log _{N}\left(\lambda_{i} / \sum_{j} \lambda_{j}\right)$.

When eigenvalues $\lambda_{i}$ of the coherence matrix $\mathbf{J}$ are given, we have for the initial Mueller matrix:

$\mathbf{M}=\sum_{q=1}^{4} \lambda_{q} \mathbf{M}_{D}^{q} ; \quad \mathbf{M}_{D}^{q} \Leftrightarrow \mathbf{T}^{q}$,

here $\mathbf{M}_{D}^{q}$ are the Mueller-Jones matrices obtained from the Jones matrices [2].

The Jones matrix, $\mathbf{T}$ (with elements $t_{i j}$ ), in turn, is obtained in the following manner:

$t_{11}^{(q)}=\tau_{1}^{(q)}+\tau_{2}^{(q)}, \quad t_{12}^{(q)}=\tau_{3}^{(q)}-i \tau_{4}^{(q)}$,

$t_{21}^{(q)}=\tau_{3}^{(q)}+i \tau_{4}^{(q)}, \quad t_{22}^{(q)}=\tau_{1}^{(q)}-\tau_{2}^{(q)}, \quad q=\overline{1,4}$,

where $\tau(q)=\left(\begin{array}{llll}\tau_{1} & \tau_{2} & \tau_{3} & \tau_{4}\end{array}\right)_{q}^{T}$ is $q$-th eigenvector of the coherence matrix $\mathbf{J}$.

Thus, the Cloude coherency matrix method is, in essence, an additive matrix model of the depolarizing Mueller matrix, and represents the initial depolarizing Mueller matrix as a weighted convex sum of four Mueller-Jones matrices.

If three of the eigenvalues of $\mathbf{J}$ vanish, then the initial matrix $\mathbf{M}$ is a deterministic Mueller-Jones matrix. If all four eigenvalues of $\mathbf{J}$ are not equal to zero and, at that, $S \leq 0.5$, then the Mueller-Jones matrix, which corresponds to the maximal eigenvalue, is the dominant type of deterministic polarization transformation of the studied medium. So, this method gives the possibility to study the anisotropy properties of depolarizing media.

In this work, we investigate the features of light depolarization by inhomogeneous anisotropic media described by the Mueller matrix Eq. (3) using the singular value depolarization metrics presented above.

\section{Simulation results and discussion}

\subsection{Cloude's coherency matrix analysis}

Cloude's coherency matrix corresponding to the Mueller matrix Eq. (3) has generally the form:

$$
\mathbf{T}=\frac{1}{2}\left(\begin{array}{cccc}
(1+b \cos (\psi)) & (a-i b \sin (\psi)) & 0 & 0 \\
(a+i b \sin (\psi)) & (1-b \cos (\psi)) & 0 & 0 \\
0 & 0 & 0 & 0 \\
0 & 0 & 0 & 0
\end{array}\right) \text {. }
$$

The eigenvalues of the coherency matrix $\mathbf{T}$ Eq. (3):

$$
\lambda_{i} \Rightarrow \frac{1}{2}\left(\begin{array}{llll}
0 & 0 & 1-\sqrt{a^{2}+b^{2}} & 1+\sqrt{a^{2}+b^{2}}
\end{array}\right) .
$$


The deterministic Mueller matrices in Cloude decomposition Eq. (11):

$$
\begin{aligned}
& \mathbf{M}_{1}=\operatorname{diag}\left(\begin{array}{llll}
1 & -1 & -1 & 1
\end{array}\right), \\
& \mathbf{M}_{2}=\operatorname{diag}\left(\begin{array}{llll}
1 & -1 & 1 & -1
\end{array}\right), \\
& \mathbf{M}_{3}=\frac{2}{a^{2}+b^{2} \sin (\psi)}\left(\begin{array}{cccc}
r & -a & 0 & 0 \\
-a & r & 0 & 0 \\
0 & 0 & -b \cos (\psi) & b \sin (\psi) \\
0 & 0 & -b \sin (\psi) & -b \cos (\psi)
\end{array}\right) \text {, } \\
& \mathbf{M}_{4}=\frac{2(r+b \cos (\psi))}{a^{2}+b^{2} \sin (\psi)}\left(\begin{array}{cccc}
r & a & 0 & 0 \\
a & r & 0 & 0 \\
0 & 0 & b \cos (\psi) & -b \sin (\psi) \\
0 & 0 & b \sin (\psi) & b \cos (\psi)
\end{array}\right),
\end{aligned}
$$

where: $r=\sqrt{a^{2}+b^{2}}$.

It can be shown that in order for Mueller matrix Eq. (3) to be physical, a following condition should be satisfied: $a^{2}+b^{2} \leq 1$. The analysis of $\mathbf{M}_{3}$ and $\mathbf{M}_{4}$ using 4-component model [10] leads us to the conclusion that these matrices are the matrices of sequence of a linear phase plate with birefringence value $\Psi$ and partial polarizer with the linear dichroism value $P$.

$$
\begin{aligned}
\mathbf{M}_{\text {LinPh }} & =\left(\begin{array}{cccc}
1 & 0 & 0 & 0 \\
0 & 1 & 0 & 0 \\
0 & 0 & \cos (\psi) & \sin (\psi) \\
0 & 0 & -\sin (\psi) & \cos (\psi)
\end{array}\right), \\
\mathbf{M}_{\text {LinAmp }} & =\left(\begin{array}{cccc}
1+P & 1-P & 0 & 0 \\
1-P & 1+P & 0 & 0 \\
0 & 0 & 2 \sqrt{P} & 0 \\
0 & 0 & 0 & 2 \sqrt{P}
\end{array}\right),
\end{aligned}
$$

then

$$
\begin{aligned}
& \mathbf{M}_{\text {LinPh. }} / \mathbf{M}_{\text {LinAmp }}= \\
& =\left(\begin{array}{cccc}
1+P & 1-P & 0 & 0 \\
1-P & 1+P & 0 & 0 \\
0 & 0 & 2 \sqrt{P} \cos (\psi) & 2 \sqrt{P} \sin (\psi) \\
0 & 0 & -2 \sqrt{P} \sin (\psi) & 2 \sqrt{P} \cos (\psi)
\end{array}\right) .
\end{aligned}
$$

For $\mathbf{M}_{3}$ we obtain:

$$
\begin{gathered}
\mathbf{M}_{3}=C_{3}\left(\begin{array}{cccc}
1 & 0 & 0 & 0 \\
0 & 1 & 0 & 0 \\
0 & 0 & \cos \left(\delta_{3}\right) & \sin \left(\delta_{3}\right) \\
0 & 0 & -\sin \left(\delta_{3}\right) & \cos \left(\delta_{3}\right)
\end{array}\right) \times \\
\times\left(\begin{array}{cccc}
1+P_{3} & 1-P_{3} & 0 & 0 \\
1-P_{3} & 1+P_{3} & 0 & 0 \\
0 & 0 & 2 \sqrt{P_{3}} & 0 \\
0 & 0 & 0 & 2 \sqrt{P_{3}}
\end{array}\right)
\end{gathered}
$$

where $\delta_{3}=\psi, P_{3}=\frac{r-a}{r+a}, \quad C_{3}=\frac{(r-b \cos (\psi))(r+a)}{a^{2}+b^{2} \sin ^{2}(\psi)}$

Using the same method for $\mathbf{M}_{4}$ :

$$
\begin{gathered}
\mathbf{M}_{4}=C_{4}\left(\begin{array}{cccc}
1 & 0 & 0 & 0 \\
0 & 1 & 0 & 0 \\
0 & 0 & \cos \left(\delta_{4}\right) & \sin \left(\delta_{4}\right) \\
0 & 0 & -\sin \left(\delta_{4}\right) & \cos \left(\delta_{4}\right)
\end{array}\right) \times \\
\times\left(\begin{array}{cccc}
1+P_{4} & 1-P_{4} & 0 & 0 \\
1-P_{4} & 1+P_{4} & 0 & 0 \\
0 & 0 & 2 \sqrt{P_{4}} & 0 \\
0 & 0 & 0 & 2 \sqrt{P_{4}}
\end{array}\right)
\end{gathered}
$$

where $\delta_{4}=-\psi, P_{4}=\frac{r+a}{r-a}, C_{4}=\frac{(r+b \cos (\psi))(r-a)}{a^{2}+b^{2} \sin ^{2}(\psi)}$.

\subsection{Singlular value depolarization metrics}

The expressions of depolarization index, polarization entropy and $Q$-metric for the Mueller matrix Eq. (3) has been determined according to [6-9] as follows:

polarization entropy

$S=-\frac{1}{2} \log _{4}\left[(1+r)^{(1+r)}(1-r)^{(1-r)}\right]$,

depolarization index

$$
\begin{gathered}
D I=\sqrt{2\left(\left(1+r^{2}\right)-1\right) / 3}, \\
Q \text {-metric }
\end{gathered}
$$$$
Q=1+2 b^{2} /\left(1+a^{2}\right) \text {. }
$$

Corresponding dependences $\left(S\left(D I, \sigma_{h}\right), S\left(Q, \sigma_{h}\right)\right.$, $S(a, b), Q(a, b))$ for the following crystals: calcite $\mathrm{CaCO}_{3}$, paratellurite $\mathrm{TeO}_{2}$ and lithium niobate $\mathrm{LiNbO}_{3}$ have been calculated and presented in (Figs 1 to 4).

Fig. 1 presents the dependences of entropy $S$ and depolarization index DI (with projections on corresponding reference planes) on the value of inhomogeneity $\sigma_{h}$. It can be seen that the minimum

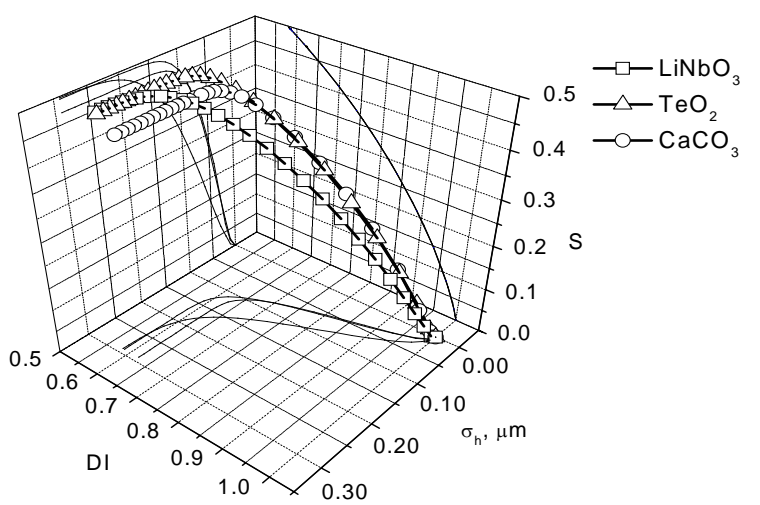

Fig. 1. Dependence of the entropy $S$ on depolarization index $D I$ and inhomogeneity $\sigma_{h}$. 


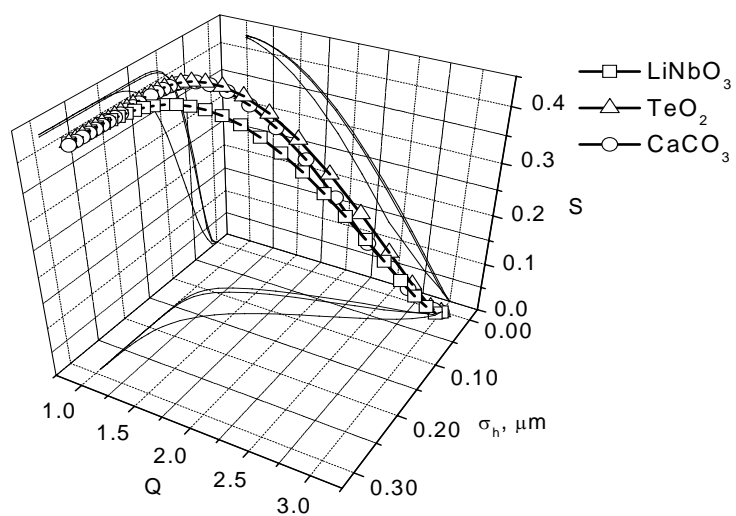

Fig. 2. Dependence of the entropy $S$ on $Q$-metric and inhomogeneity $\sigma_{h}$.

value of depolarization index and the maximum value of entropy are various for different crystals. This, as it will be illustrated below, is determined by the values of refractive indexes of crystals.

Fig. 2 shows that the maximum value of $Q_{\max }=3$ is observed at $\sigma_{h}=0$ for all the crystals, i.e., in the case when the Mueller matrix Eq. (3) corresponds to an ordinary linear birefringent plate. The minimum value is equal for all the crystals as well as for $Q_{\min }=1$, which corresponds to the case of partially depolarizing medium.

From Figs 3 and 4, one can see that the minimum value of $Q$-metric and maximum value of entropy $S$ are reached at different values of $a$ for different crystals, Figs $3 \mathrm{~b}$ and $4 \mathrm{~b}$. This is determined by the values of refractive indexes of the crystals. Indeed, in case of large inhomogeneity $\left(\sigma_{h} \rightarrow \infty\right)$, we have:

$a_{\sigma_{h} \rightarrow \infty}=\frac{\left(n_{2}-1\right)^{2}-\left(n_{1}-1\right)^{2}}{\left(n_{2}-1\right)^{2}+\left(n_{1}-1\right)^{2}}=\frac{1-\xi^{2}}{1+\xi^{2}}$,

$b_{\sigma_{h} \rightarrow \infty}=0$,

where $\xi$ is of the form:

$\xi=\left(n_{1}-1\right) /\left(n_{2}-1\right)$.

Thus, the entropy $S$ for this case is

$S_{\sigma_{h} \rightarrow \infty}=-\log _{4}\left(\left(\frac{1}{1+\xi^{2}}\right)^{\left(\frac{1}{1+\xi^{2}}\right)}\left(\frac{1}{1+\frac{1}{\xi^{2}}}\right)^{\left(\frac{1}{1+\frac{1}{\xi^{2}}}\right)}\right)$.

Corresponding numerical values of $\xi, a_{\sigma_{h} \rightarrow \infty}$, $S_{\sigma_{h} \rightarrow \infty}$ and value of inhomogeneity $\sigma_{h}$ when in matrix Eq. (3) $b=0$, i.e. when the case of large inhomogeneity takes place, are presented in the table.
Table. Numerical values of $\xi, a_{\sigma_{h} \rightarrow \infty}, S_{\sigma_{h} \rightarrow \infty}$ and $\sigma_{h}$ for the case of large inhomogeneity.

\begin{tabular}{|c|c|c|c|c|c|c|}
\hline & $n_{1}$ & $n_{2}$ & $\xi$ & $a_{\sigma_{h} \rightarrow \infty}$ & $S_{\sigma_{h} \rightarrow \infty}$ & $\begin{array}{c}\sigma_{h}, \\
\mu \mathrm{m}\end{array}$ \\
\hline $\mathrm{LiNbO}_{3}$ & 2.208 & 2.300 & 0.93 & 0.07 & 0.498 & 2.896 \\
\hline $\mathrm{TeO}_{2}$ & 2.259 & 2.411 & 0.89 & 0.11 & 0.495 & 1.753 \\
\hline $\mathrm{CaCO}_{3}$ & 1.489 & 1.655 & 0.75 & 0.28 & 0.470 & 1.605 \\
\hline
\end{tabular}

It needs to note that $S_{\sigma_{h} \rightarrow \infty}$ in Eq. (30) is equal 0.5 when $\xi=1$. This means that $n_{1}=n_{2}$ in this case and, thus, $S_{\sigma_{h} \rightarrow \infty}$ for matrix Eq. (3) can never be equal to 0.5 .
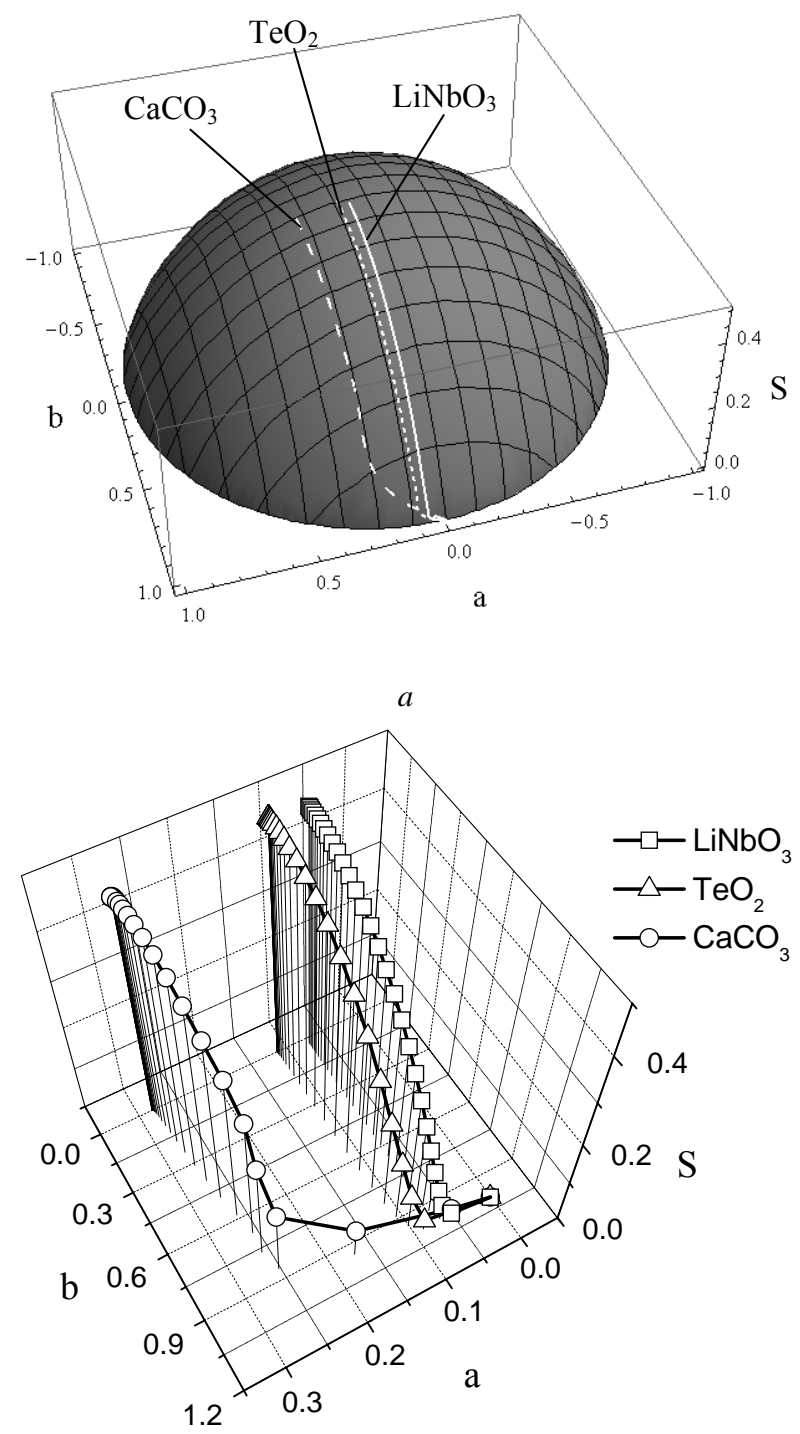

$b$

Fig. 3. Dependence of the entropy $S$, Eq. (24), as a function of $a$ and $b$. 

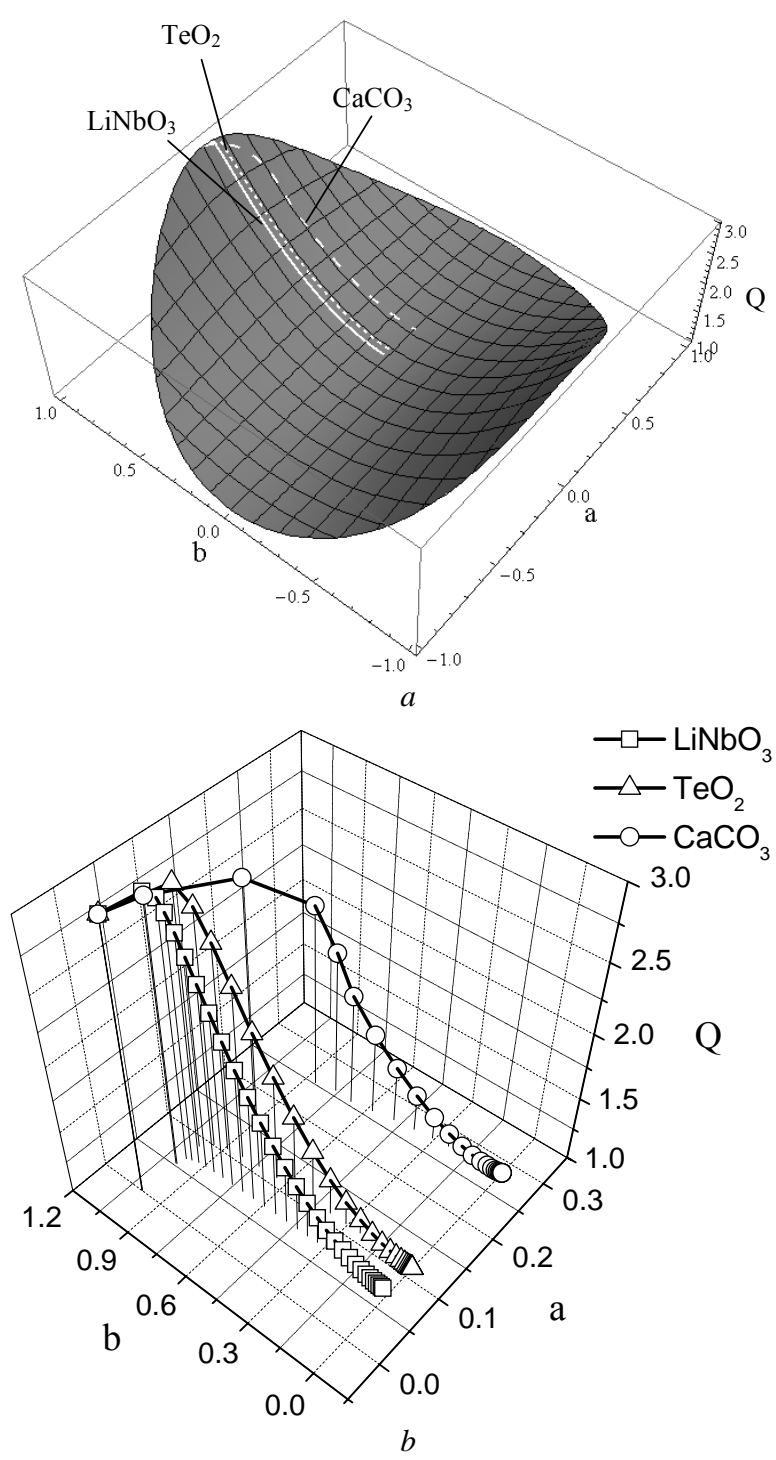

Fig. 4. Dependence of the $Q$-metric, Eq. (26), as a function of $a$ and $b$.

Dependences from Figs $3 b$ and $4 b$ for calcite, paratellurite and lithium niobate are presented in Figs 3a and $4 \mathrm{a}$ by white lines. Surfaces in Figs $3 \mathrm{a}$ and $4 \mathrm{a}$ determine all the possible values of $Q$-metric and entropy $S$ as functions of $a$ and $b$ for the media described by the Mueller matrix model Eq. (3).

\section{Conclusions}

In summary, we have studied depolarization of light by inhomogeneous anisotropic lossless crystalline medium in the single scattering case. We describe this medium with the depolarization index $D I, Q$-metric and the polarization entropy $S$ that is added by the medium to the scattered field. These quantities provide insights into the particular depolarization mechanisms of the various media. It has been shown that the polarization entropy of scattering medium in the case of large inhomogeneity differs from its maximal possible value for the class of depolarizing media with only two non-zero coherency matrix eigenvalues $S_{\max }=0.5$.

\section{References}

1. S.N. Savenkov, R.S. Muttiah, K.E. Yushtin, S.A. Volchkov, Mueller matrix model of inhomogeneous, linear, birefringent medium: single scattering case // Journal of Quantitative Spectroscopy \& Radiative Transfer 106, p. 475-486 (2007).

2. Ch. Brosseau, Fundamentals of Polarized Light. A Statistical Optics Approach. New York, NorthHolland Publishing Company, p. 406, 1998.

3. P. Huard, Polarization of Light. New York, Wiley, p. 320, 1997.

4. A.A. Kokhanovsky, Light Scattering Media Optics: Problems and Solutions. Chichester, Praxis Publishing, p. 403, 2001.

5. S.N. Savenkov, Scattering (Mueller) matrices and experimental determination of matrix elements, Chap. 4, In: From Laboratory Spectroscopy to Remotely Sensed Spectra of Terrestrial Ecosystems, Eds. R.S. Muttiah. Kluwer Academic Publishers, Dordrecht, The Netherlands, p. 85-107, 2002.

6. J.J. Gil, E. Bernabeu, Depolarization and polarization indexes of an optical system // Opt. Acta 33, p. 185-189 (1986).

7. R. Espinosa-Luna, E. Bernabeu, On the Q(M) depolarization metric // Opt. Communs 277, p. 256258 (2007).

8. S.R. Cloude, Group theory and polarization algebra // Optik (Stuttgart) 7, p. 26-36 (1986).

9. S.R. Cloude, E. Pottier, Concept of polarization entropy in optical scattering // Opt. Eng. 34, p. 1599-1610 (1995)

10. S.N. Savenkov, V.V. Marienko, E.A. Oberemok, O.I. Sydoruk, Generalized matrix equivalence theorem for polarization theory // Phys. Rev. E 74, 056607 (2006). 